N93-2013?

\title{
LASER-INITIATED ORDNANCE FOR \\ AIR-TO-AIR MISSILES
}

Presented by: David R. Sumpter

McDonnell Douglas Missile Systems Company

Approved for public release; distribution is unlimited. 


\title{
LASER-INITIATED ORDNANCE FOR AIR-TO-AIR MISSILES
}

\begin{abstract}
McDonnell Douglas Missile Systems Company (MDMSC) has developed a Laser Ignition Subsystem (LIS) for Air-to-Air missile applications. The MDMSC subsystem is designed to activate batteries, unlock fins, and sequence propulsion system events. The subsystem includes Pyro Zirconium Pump (PZP) lasers, mechanical Safe \& Arm, fiber-optic distribution system, and optically activated pyrotechnic devices (initiators, detonators and thermal batteries). The LIS design has incorporated testability features for the laser modules, drive electronics, fiber-optics, and pyrotechnics. Several of the LIS have been fabricated; and have supported thermal battery testing, integral rocket ramjet testing, and have been integrated into integral rocket ramjet flight test vehicles as part of the flight control subsystem.
\end{abstract}

\section{Introduction}

McDonnell Douglas Missile Systems Company, with Hercules/Allegany Ballistics Laboratory, SAFT America, and Hi Shear has demonstrated the use of laserinitiated pyrotechnics to sequence events in the Advanced Air-to-Air Missile (AAAM) D\&V program. The Laser Ignition Subsystem (LIS) uses six Pyro-Zirconium Pump (PZP) lasers to initiate the thirteen pyrotechnics, controlling the activation of the Fin Unlock, Battery Activate, and Integral Rocket Ramjet functions. Two distinct initiators and a detonator were developed for the LIS, along with a unique activation train within the thermal batteries. The lasers and associated control and test functions were housed within two modules, which are form/fit compatible with the AAAM application. Each of the six laser modules require Arm and Fire signals for activation, and feature a mechanical Safe/Arm device to preclude inadvertent firing of the lasers and pyrotechnics. The fiber optic harness incorporates multiple strands of $200 \mu \mathrm{m}$ and 400 $\mu \mathrm{m}$ fibers routed to each pyrotechnic. Built-In Test features of the system include optical integrity checks from laser rod to pyrotechnic, lamp module status (fired/unfired), and fire function monitor.

The LIS has proven to be reliable, and has been successfully used in the development of the thermal batteries, booster and ramjet sustainer. It has been subjected to strenuous environmental tests and retained operational capability. Several have been installed the the AAAM Air Vehicles in preparation for flight demonstration.

\section{Integral Rocket Ramjet System}

The Integral Rocket Ramjet (IRR) system concept applied to the AAAM program is outlined in Figure 1. The Flight Controls regulates all activity in the system. It transmits Arm and Fire commands to the LIS to activate the thermal batteries, unlock the fins, and sequence the booster and sustainer functions. The Flight Controls also commands fin position and manages the fuel flow to control vehicle flight.

There are six laser modules in the LIS, which are fired in response to commands from the Flight Controls. The first event is the activation of the two thermal batteries, which is performed using aircraft power prior to launch. The second laser is 


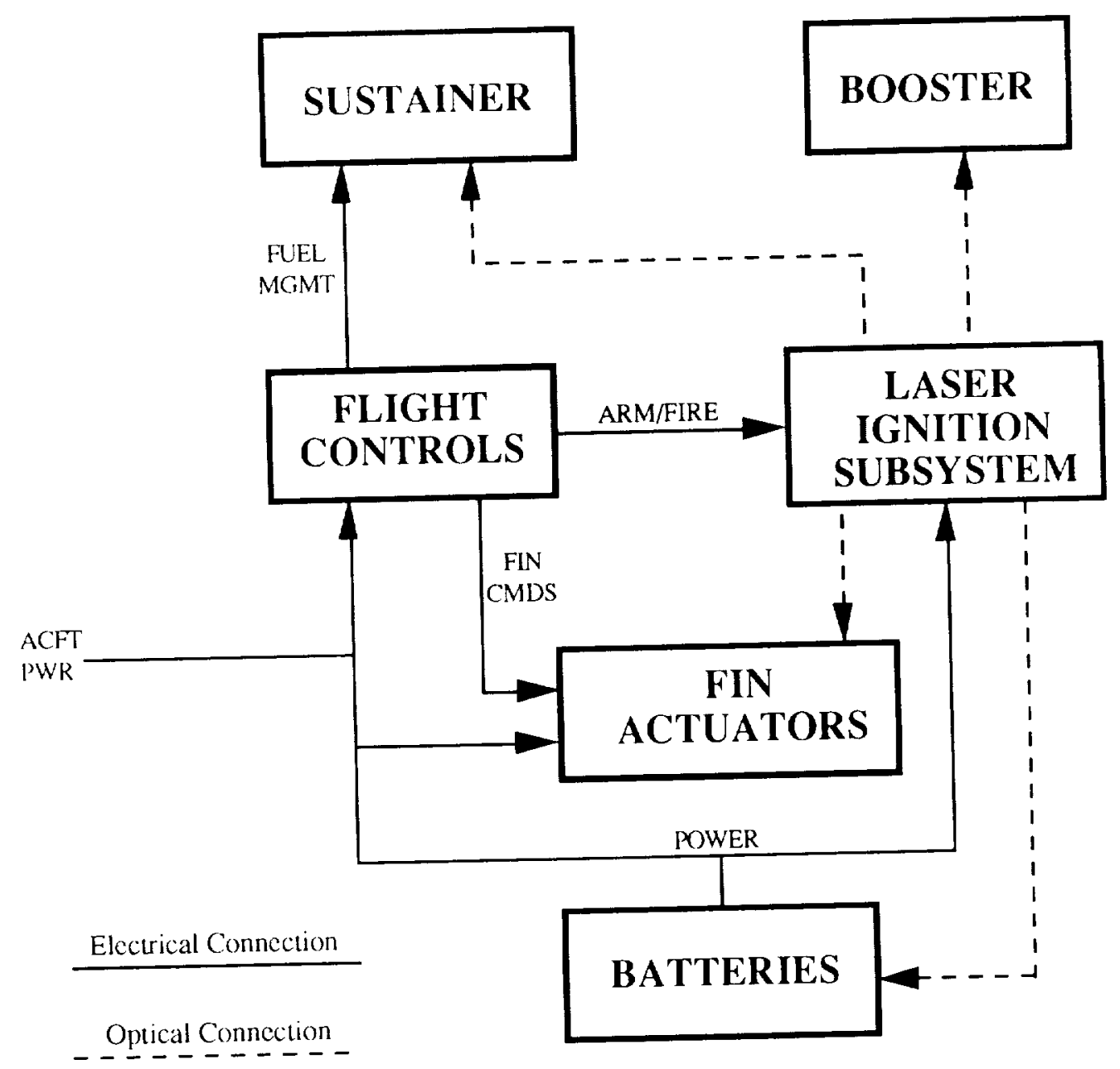

FIGURE 1. Integral Rocket Ramjet System Concept

used to unlock the four fins simultaneously. The remaining four lasers are used to sequence the propulsion events, which use a total of seven pyrotechnic devices. Each of the six lasers is fired only once, activating a total of 13 pyrotechnics.

\section{The LIS Control Assemblies}

The lasers, Safe/Arm devices, and associated electronics are packaged in two housings, as shown in Figure 2. The port assembly consists of the laser modules for the Battery Activate and Fin Unlock, and has the Optical BIT detector circuitry for all laser functions. The starboard assembly contains the laser modules for the propulsion events.

The segregation of the events in this manner is not merely for convenience. Only the battery power is routed to the starboard control assembly, significantly reducing the risk of an inadvertent firing before launch. The port assembly requires a single Arm command to arm both lasers, and a distinct fire command to activate the specific lasers. For safety purposes, the starboard assembly requires two Arm commands to arm all four lasers, and then the distinct fire commands to activate the specific lasers. Neither starboard Arm command is shared by the port assembly, and 
the two commands are generated by separate sources to further preclude a failureinduced ignition.

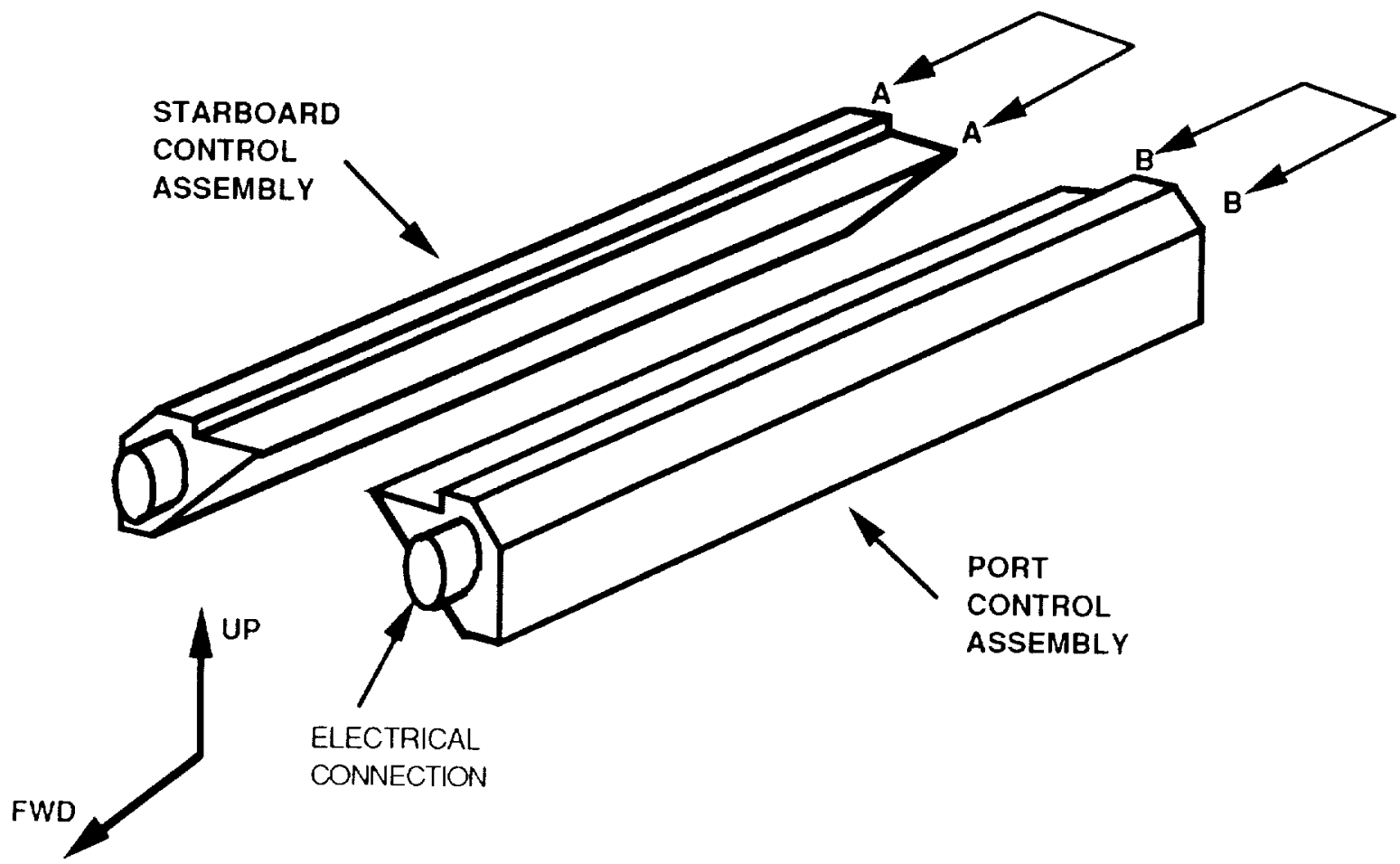

VIEW B-B

PORT CONTROL ASSEMBLY

VIEW A-A

STARBOARD CONTROL ASSEMBLY
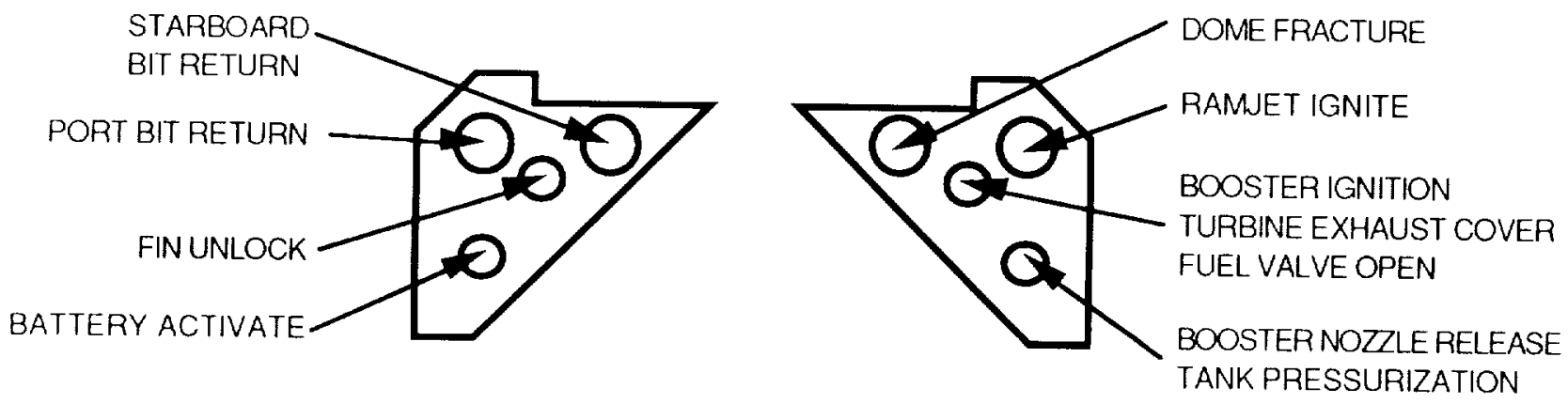

FIGURE 2. Laser Ignition Subsystem (LIS) Control Assemblies

The laser sources are based on a PZP system. Five flashlamps are used to excite the Neodynium-Glass laser rod, which generates a 2 Joule pulse at $1060 \mathrm{~nm}$. As shown in Figure 3, this energy passes through a focusing lens to reduce the beam size for the fiber optic cable. In the Safe mode, a shutter interrupts the optical path, using a dichroic-coated window to block the laser energy. When Armed, the window is mechanically removed from the beam path, permitting the laser energy to pass into the fiber optics. A short length of $1000 \mu \mathrm{m}$ fiber is used as an environmental seal for the control assemblies. When the fiber optic cable is connected, the laser energy is transmitted through the cable to the pyrotechnic device. 


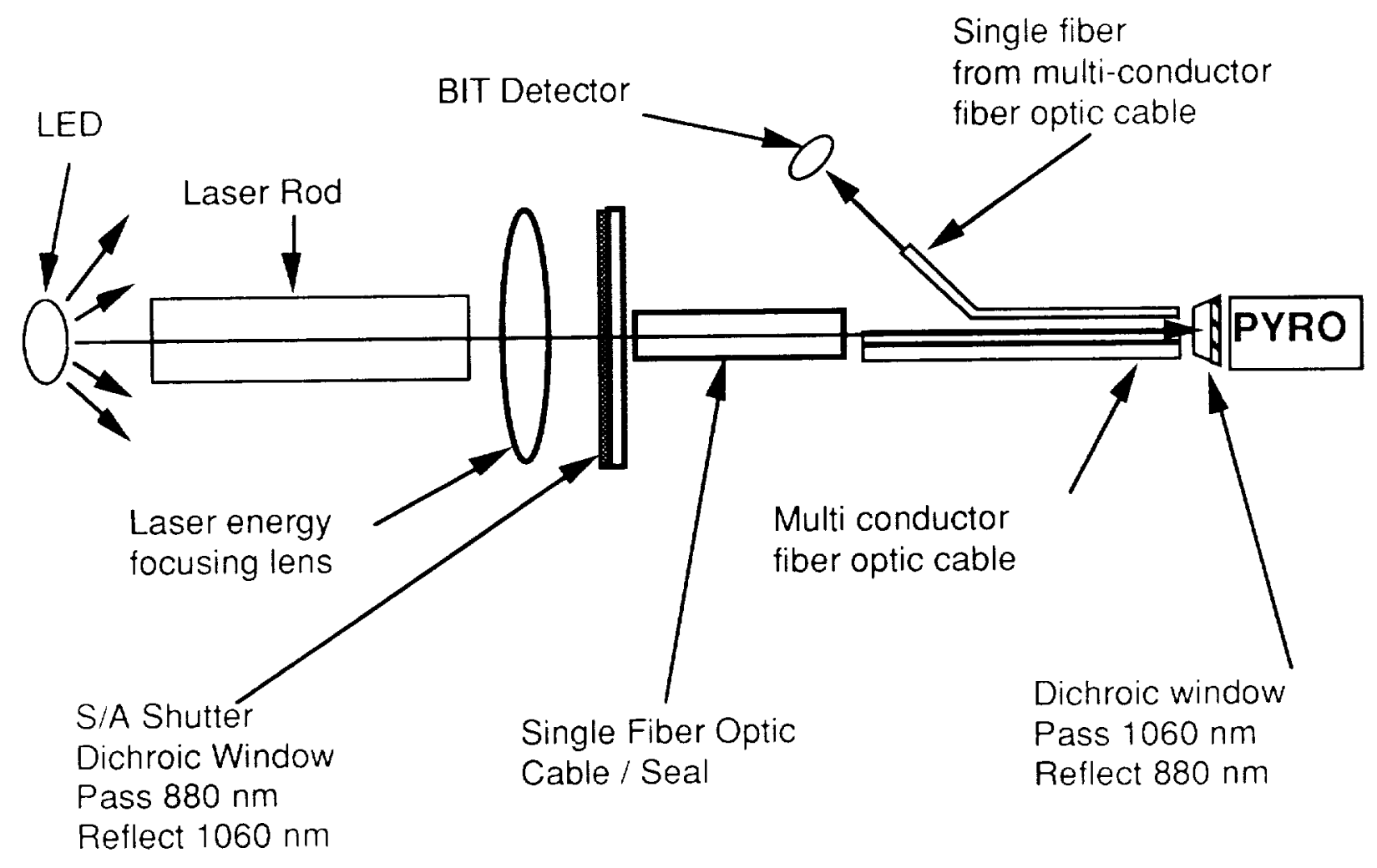

FIGURE 3. Optical Path for Laser and BIT Energy

The laser modules are grouped in pairs, and share a rotary solenoid for the Safe/Arm function. In the Safe mode, the rotary solenoid shorts the flashlamps to ground, and positions the shutter to interrupt the optical path. When armed, the flashlamps are connected to the fire circuii, and the shutter is removed from the optical path. Figure 4 shows a pair of laser modules with the common Safe/Arm device.

There are three Built-In Test (BIT) functions in the LIS. The first uses a metalized film wrapped around the flashlamps to determine flashlamp integrity. When the flashlamps are fired, the metalized film melts, changing a low-impedance path to high impedance. Sensing the impedance flags expended laser modules. The second test checks the fire circuitry. When the fire circuit is activated, the Optical BIT function is inhibited. A failed Optical BIT during a fire command, when preceded and succeeded by a passing Optical BIT, verifies the performance of the fire circuits.

The third BIT function is Optical BIT. The integrity of the optical path, from laser rod to pyrotechnic is verified by this function. The Optical BIT concept is shown in Figure 3. An LED is positioned behind the laser rod. The 880 nanometer radiation propagates through the laser rod and focusing lens. The position of the shutter does not seriously impact the function, since the dichroic passes greater than $90 \%$ of the BIT energy, and is out of the path when armed. The BIT energy passes out of the control assemblies, through the fiber optic cables, to the pyrotechnics. The dichroic element 
In the pyrotechnic is reflective to the $880 \mathrm{~nm}$ energy. A single fiber, dedicated to Optical BIT, originating at the pyrotechnic termination, collects the reflected BIT energy and transmits it back to a detector in the control assembly. The detectors are monitored to assess the status of the optical path.

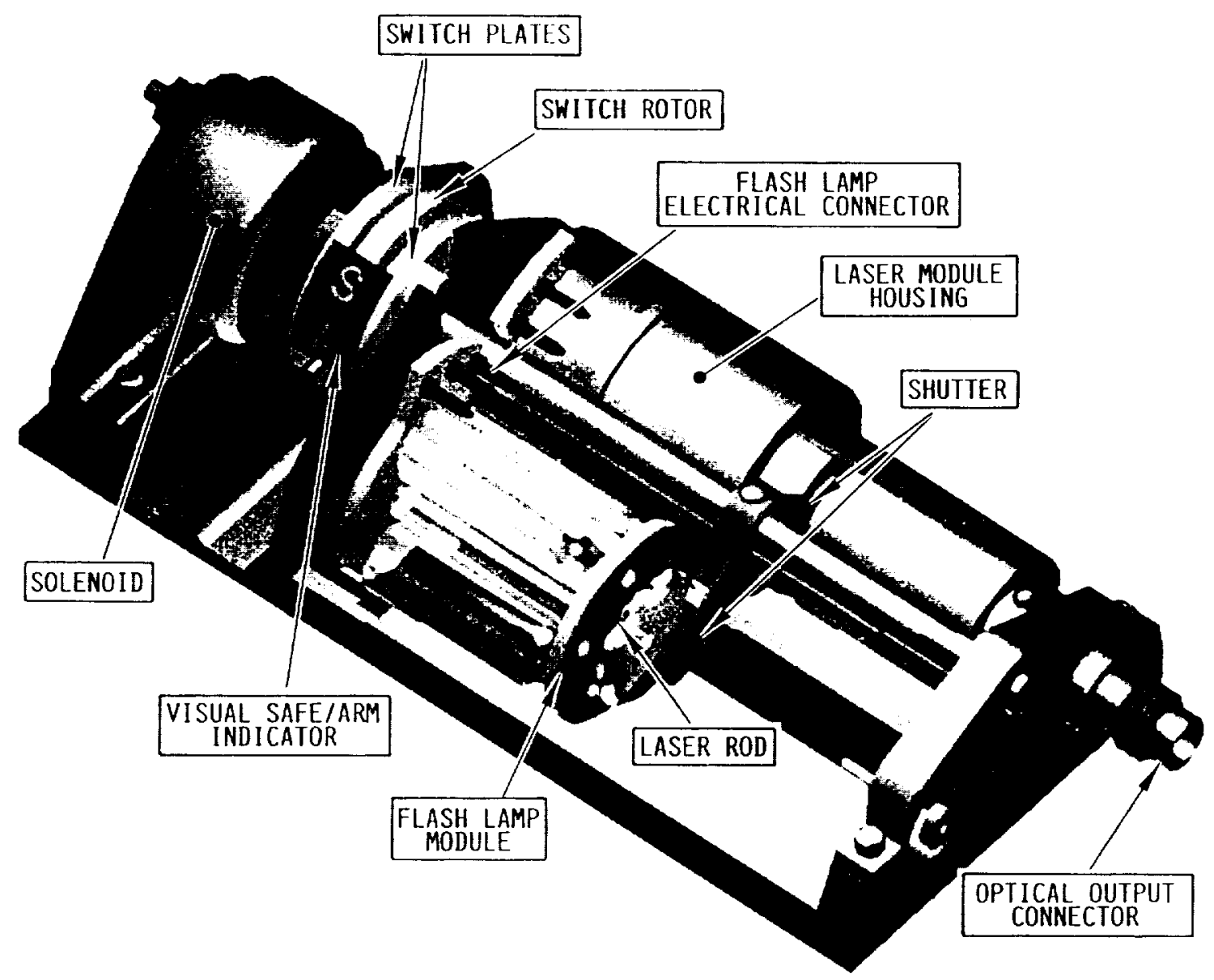

FIGURE 4. A Two-Pulse Laser Assembly

\section{Initiators and Detonators}

The LIS uses six initiator and and detonator designs for the Integral Rocket Ramjet concept. The basic, or "standard" initiator, is shown in Figure 5. The fiber optic termination uses a conventional SMA connector. The pin seats on the outer rim of the receptacle, and is polished to a tightly controlled length to maintain a minimal air gap between the pin and the window. The glass window is tapered to provide a seating surface, and has a dichroic coating on the inner surface (adjacent to the propellant). This dichroic coating passes the laser energy, but reflects the Optical BIT energy back into the fiber optic for detection. The propellant consists of a Zirconium Potassium Perchlorate (ZPP) donor charge, and $\mathrm{BKNO}_{3}$ as the primary propellant. A spacer occupies the extra volume, holding the propellant in position. 


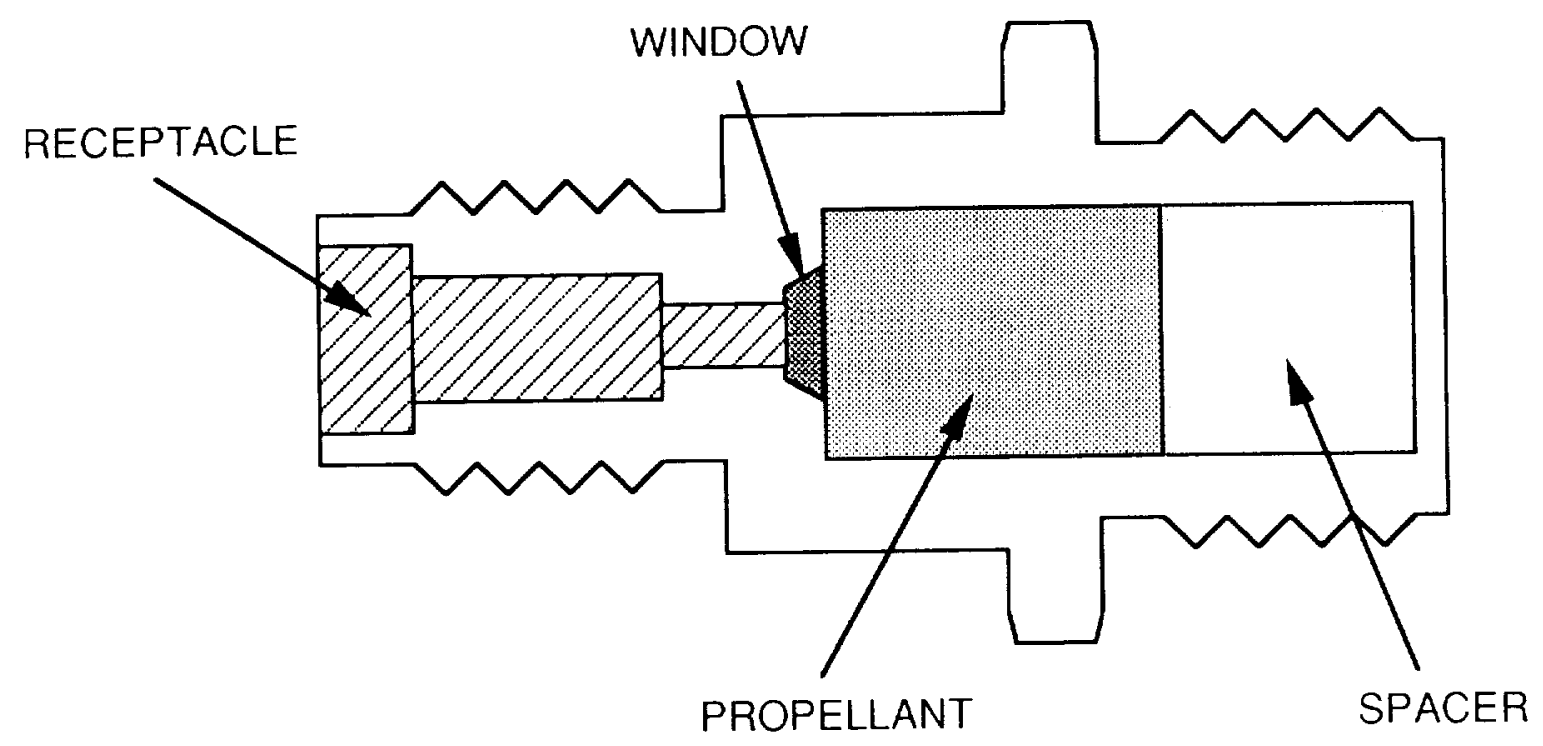

FIGURE 5. Standard Initiator Configuration

There are three variations of this initiator. Two differ only in the amount of propellant, since two different output pressures were needed. The third does not use the SMA optical interface. This third device is the Fin Unlock Initiator, which is installed in the removable fins. The fiber optic is terminated in the fin control actuator, and is spring-loaded to maintain contact with the initiator when a fin is installed. The Fin Unlock Initiator looks like the standard initiator with the SMA connection removed, as shown in Figure 6. To accept the blind mate of the fiber optic pin to the initiator, the receptacle is chamfered.

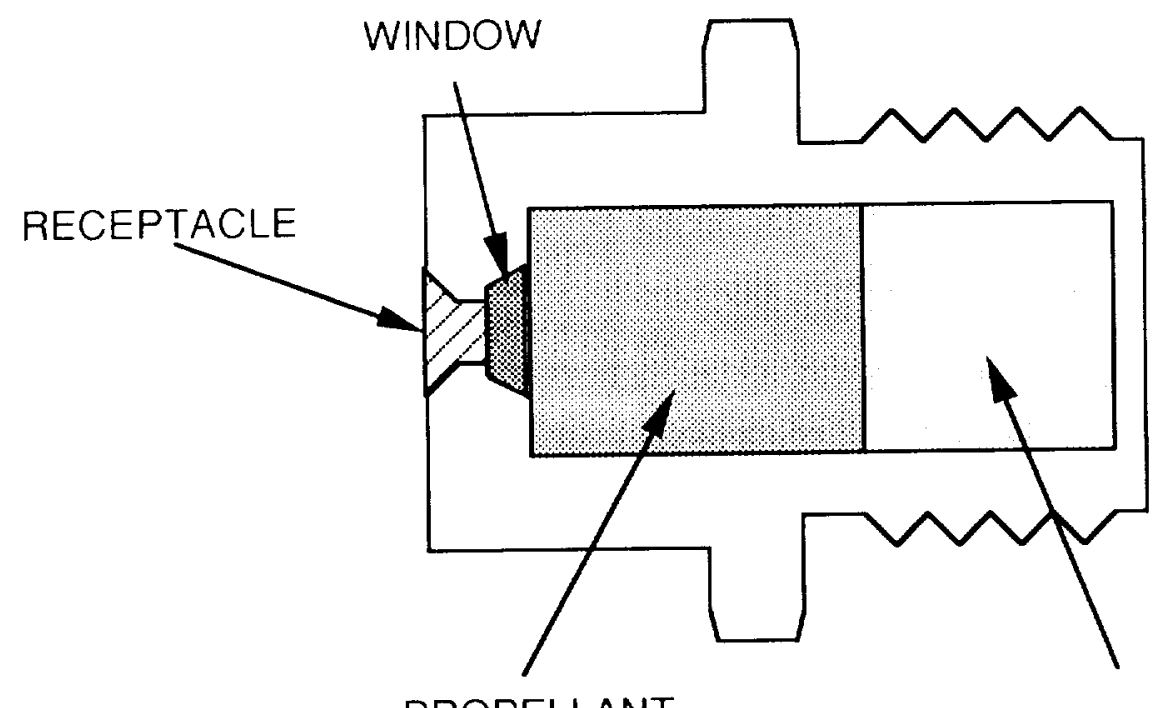

SPACER

FIGURE 6. Fin Unlock Initiator Configuration 
The detonator design is also very similar to the standard initiator. The optical interface is the same, however the charge is different. A small amount of ZPP is used to accept the laser energy. Instead of directly igniting the propellant of the initiator, the ZPP in the detonator ignites a donor charge of PETN, which in turn ignites the primary PETN explosive charge. The detonator does not need the spacer, since the entire charge cavity is utilized.

The remaining two initiators are unique devices. The first is used to ignite the booster. The booster igniter must operate radially in the booster port, instead of an axial output like the standard initiator. The approach used was a bag of $\mathrm{BKNO}_{3}$ pellets embedded in the booster port. The initiator is placed in the bag of pellets, which is then installed in the booster port and foamed in place. Since this device is installed as part of the booster manufacturing and is not directly accessible, a fiber optic lead is required as part of the delivered booster. Figure 7 shows the configuration of the booster initiator.

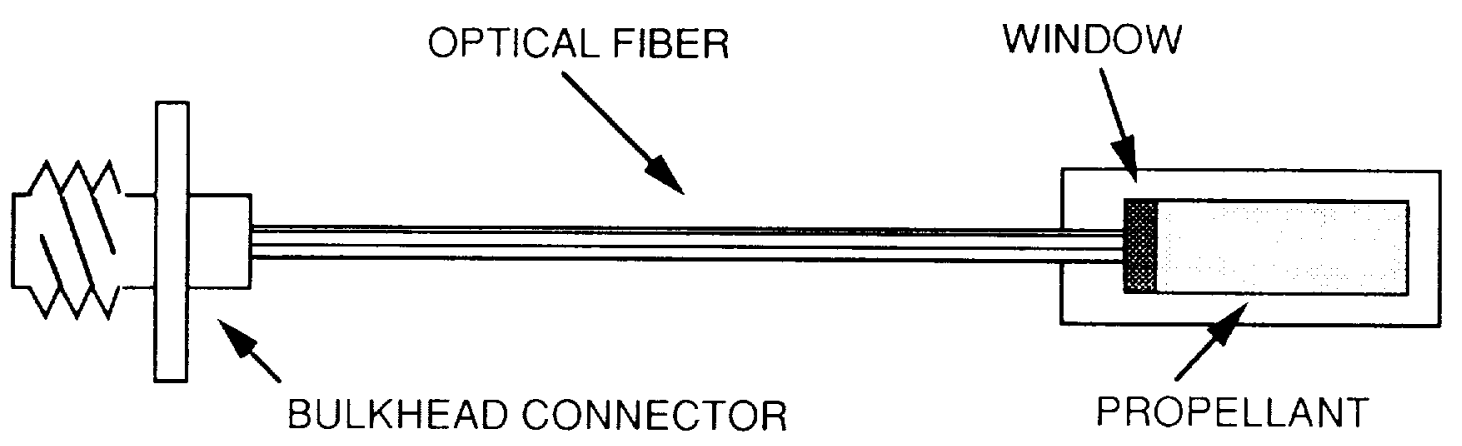

FIGURE 7. Booster Initiator Configuration

The bulkhead connector interface is the standard SMA contact. The bundle of optical fibers lead from the bulkhead connector to the initiator, and are terminated at the glass window. Like the other devices, the window has a dichroic on the second surface to reflect the Optical BIT energy, and pass the laser energy. The propellant consists of a small donor charge of ZPP, with a primary charge of $\mathrm{BKNO}_{3}$. The body of the initiator is nylon which is sealed with epoxy, permitting the propellant to rupture it easily to activate the booster igniter.

The final initiator type is built into the thermal batteries. The configuration is illustrated in Figure 8 . The receptacle is a standard SMA connection, and the glass window is the same as that used for the standard initiator, including the dichroic coating. The donor charge is again ZPP. The ZPP ignites the fuze, which extends through the core of the battery. The fuze then activates the cells in the battery stack.

Note that the dichroic window on each of the devices is on the inside of the device. This was done for two reasons. First, it provides a gap between the polished pin and the reflective surface, which is needed to scatter the Optical BIT energy from the transmit fibers to the single return fiber. Second, it is burned off when the device is activated, which will cause Optical BIT to fail, flagging an expended device. 


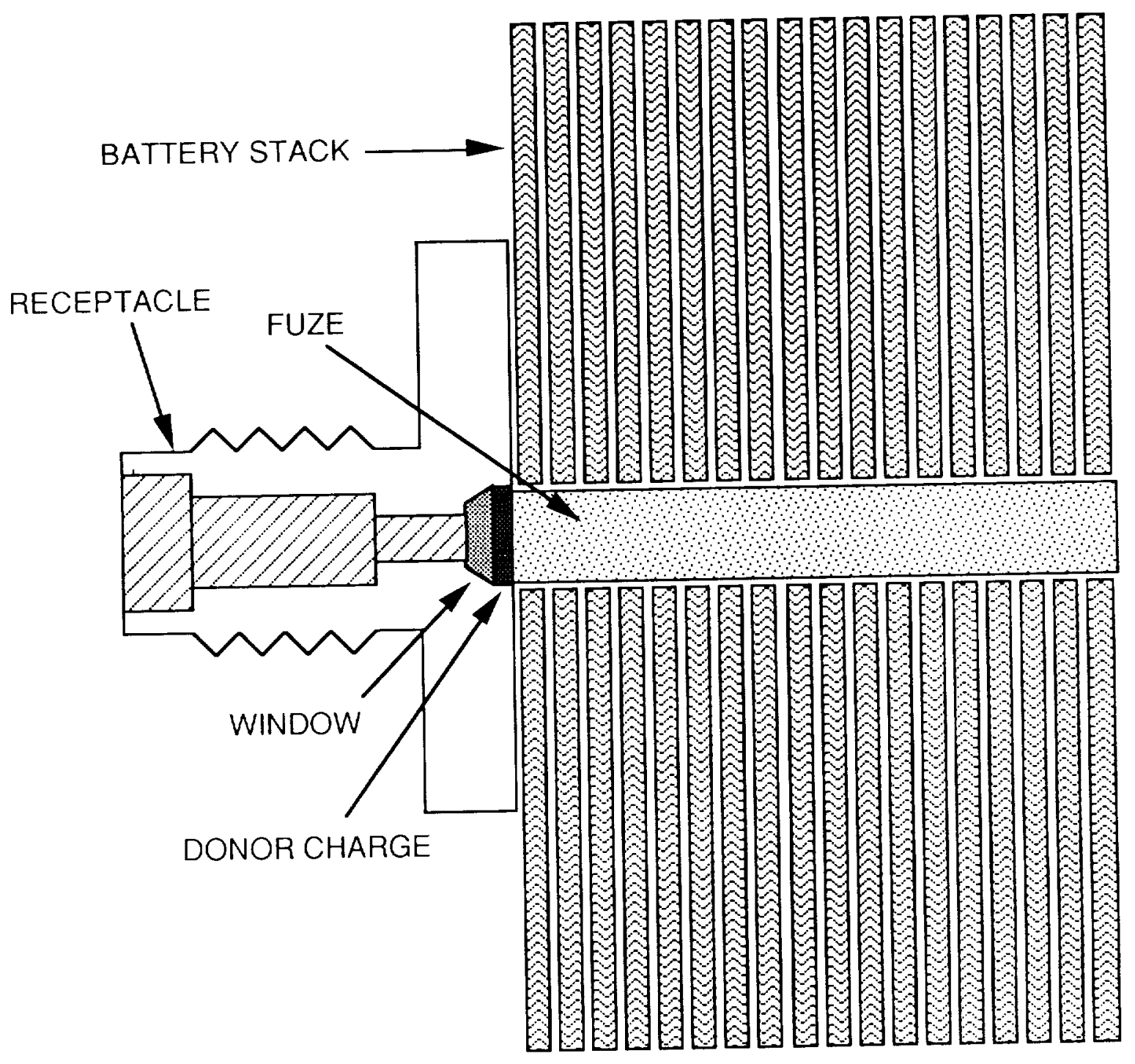

FIGURE 8. Battery Initiator Configuration

\section{Fiber Optics}

The fiber optics are used to transmit the Optical BIT and laser energy to the pyrotechnic devices, and return the reflected Optical BIT energy to the detectors. Each fiber optic has multiple transmit fibers and a single return fiber routed to each pyrotechnic device. The most complicated is the Fin Unlock, which is shown in Figure 9.

The Fin Unlock fiber optic cable starts at the Control Assembly with nineteen fibers. The number nineteen is significant because it produces a stable pattern, with one in the center, an inner ring of six, and an outer ring of twelve fibers. These nineteen fibers are divided between the four fin unlocks. The primary consideration is the power density of the laser beam, which is highest in the center, and falls off rapidly at the outside of the beam. The four groups of fibers are selected such that each fin unlock will receive approximately the same laser power. 


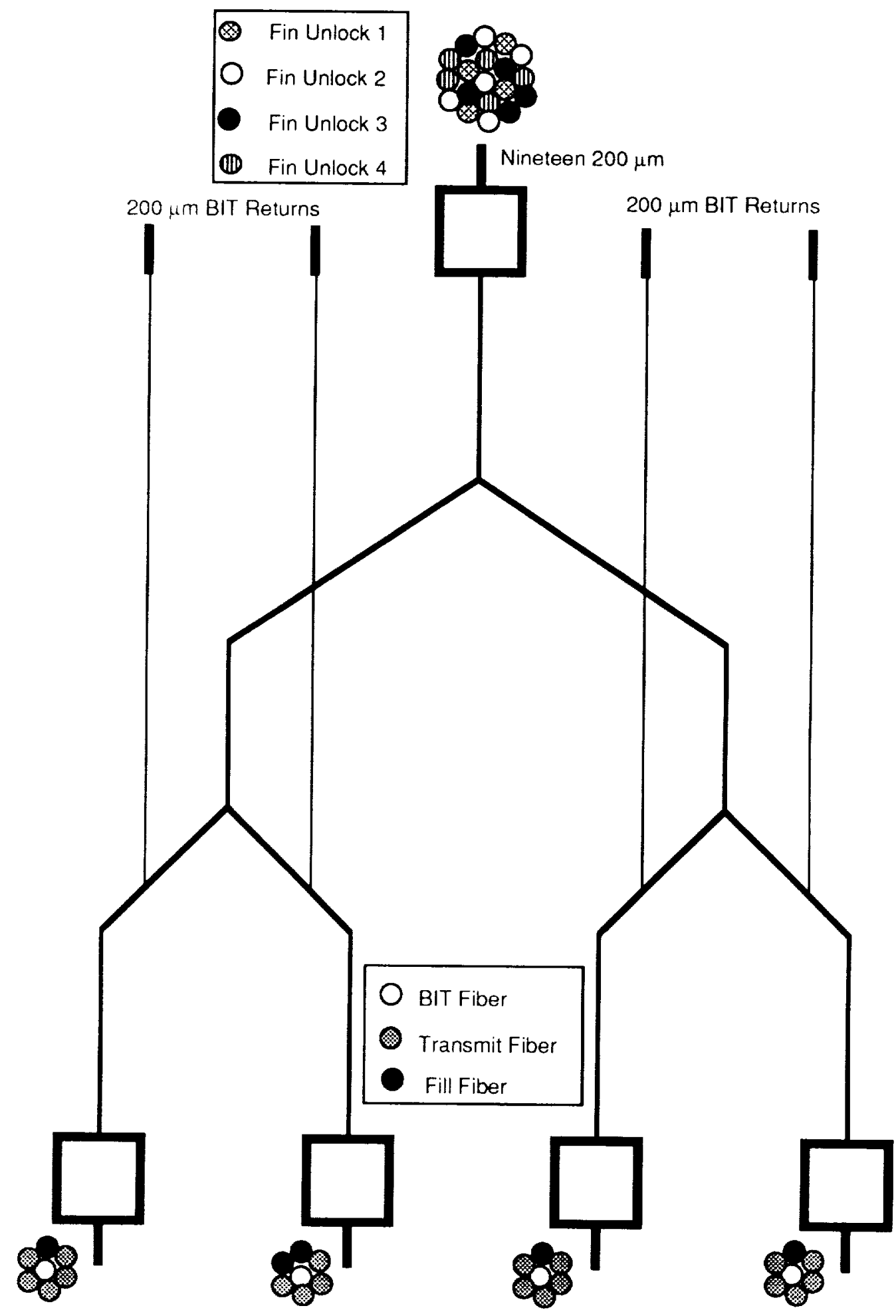

FIGURE 9. Fin Unlock Fiber Optic 
The four groups of fibers are separated into individual cable branches, and routed to the four fin unlocks. Note that three of the fin unlocks have five transmit fibers, the other has only four. At the fin unlock terminations, the stable pattern size is now seven: the center fiber and the ring of six surrounding it. The center fiber is always the Optical BIT return fiber, so that it can collect scattered BIT energy from all adjacent fibers. Since none of the four fin unlock terminations has all seven fibers assigned, fill fibers are inserted to complete the stable pattern. These fibers are cut off in the cable just outside of the pin, and serve no purpose other than filling the gaps.

The individual Optical BIT return fibers are routed to the port control assembly, where all the BIT detectors are located. The port control assembly has two connectors of seven pins (one pin is unused) for the BIT returns. Each pin is inserted into the assigned position, then all are held in place with an insert and nut.

The five remaining fiber optic cables are designed using the same considerations. The cables which activate two or less devices use 400 micron fiber for the transmit fibers instead of the 200 micron, since fewer 400 micron fibers can carry the same energy, and the energy division problem is simpler. When the 400 micron fiber is used, the stable patterns used are seven and four fiber bundles.

\section{Test Results}

The LIS has been subjected to thermal, vibration and shock testing using the AAAM environments. The dynamic environments are reasonably severe. Operation has been demonstrated during and after $19 \mathrm{Grms}$ vibration, and after $42 \mathrm{G}$ shock. The temperature environments are not as severe; operation has been demonstrated during and after $+160^{\circ} \mathrm{F}$ and $-65^{\circ} \mathrm{F}$ soaks.

The LIS has also been used to develop other systems for the Integral Rocket Ramjet. The thermal batteries, fin unlocks, booster and ramjet sustainer functions have all been demonstrated. This testing has shown the fiber optics to be unreliable after repeated use. When firing the laser through the fiber optic bundles, some of the laser energy is absorbed by the epoxy between the fibers. This localized heating causes the epoxy to boil, which results in contamination being spattered on the polished fiber optic surface. Attempt to re-use the fiber optic, without additional polishing to clean the surface, results in attenuation which can be severe enough that the energy reaching the pyrotechnic is below the all-fire level. This condition is detectable by Optical BIT, however BIT was not used for the development tests. Also note that this condition does not impact a deployed system, where the fiber optics are only used once.

\section{Summary}

A laser ignition system has been developed and demonstrated for the Integral Rocket Ramjet concept applicable to Air-to-Air missiles. The design has been used to support development and testing of other subsystems. Several of the LIS have been installed in air vehicles. 
This page intentionally left blank. 